\title{
Background level estimations using Pulse Shape Analysis in $\mathrm{Ge}$ experiments
}

\section{H. Gómez ${ }^{1}$}

University of Zaragoza

Pedro Cerbuna 12, 50009 Zaragoza - SPAIN

E-mail: hgomez@unizar.es

\section{S. Cebrián}

University of Zaragoza

Pedro Cerbuna 12, 50009 Zaragoza - SPAIN

E-mail: scebrian@unizar.es

\section{J. Morales}

University of Zaragoza

Pedro Cerbuna 12, 50009 Zaragoza - SPAIN

E-mail: jmorales@unizar.es

\section{J. A. Villar}

University of Zaragoza

Pedro Cerbuna 12, 50009 Zaragoza - SPAIN

E-mail:villar@unizar.es

Some neutrino properties, like its mass, could be determined or at least delimited using germanium detectors enriched in ${ }^{76} \mathrm{Ge}$ and studying the neutrinoless double beta decay of this isotope. Furthermore this kind of detectors can also be used for detection of Dark Matter exploring the low energy region.

In order to have the sensitivity required, the background level should be around $10^{-3}$ $\mathrm{c} / \mathrm{keV} / \mathrm{kg} / \mathrm{y}$ in the region of interest for the neutrinoless double beta decay signal. Some previous studies showed that rejection of background events using Pulse Shape Analysis (PSA) can be a very powerful tool to reach this level offering high efficiency for neutrinoless double beta decay events detection. To determine the rejection capability of this method and the detection efficiency, a set of simulations were developed showing that three-dimensional spatial resolution and not only in radial direction, is necessary in order to reject enough multi-site events to reach the required background level.

Identification of dark matter 2008

Stockholm, Sweden

August 18-22, 2008

\footnotetext{
$1 \quad$ Speaker
} 


\section{Introduction}

New generation of neutrinoless double beta decay experiments using ${ }^{76} \mathrm{Ge}$ enriched germanium detectors, aim to reach enough sensitivity to explore effective neutrino masses around $40 \mathrm{meV}$. One of the requirements to reach this sensitivity is to reduce the background level down to $10^{-3} \mathrm{c} / \mathrm{keV} / \mathrm{kg} / \mathrm{y}$.

Several techniques to reach this goal could be considered: granularity of the experiment, segmentation of the detectors and further application of anticoincidence techniques or PSA are three of the techniques that should be used alone or together with the others. Recent simulations showed that PSA in segmented detectors seems to be the best technique to reject background events giving in addition the highest values for neutrinoless double beta decay events detection efficiency [1].

For these reasons, a numerical evaluation of the background level that could be reached using the PSA is necessary to see if it is low enough for the expected sensitivity, and has been attempted in this work. Procedures to generate and analyze the electric pulses, results and conclusions will be presented in the following sections.

\section{Development features}

Since electric pulses are generated due to the collection in the detector electrodes of the charge carriers produced by an energy deposit, it is necessary to have a background events library. An application based on the GEANT4 [2] simulation package provides the energy value and position of these events coming from the main background sources: internal ${ }^{60} \mathrm{Co}$ and ${ }^{68} \mathrm{Ge}$ due to the cosmogenic activation of the crystal [3], external ${ }^{208} \mathrm{Tl}$ and ${ }^{214} \mathrm{Bi}$ contamination and ${ }^{60} \mathrm{Co}$ contamination from copper cryostat and other components close to the detector. All these sources are very important because they have radioactive emissions above the experiment 2.0-2.1 MeV region of interest (RoI).

Electric pulses are generated for a true coaxial detector with different masses and segmentation schemes. In all the cases, a positive potential is applied in the outer contact while the inner one is at ground. The electric field has been calculated using finite element calculation software [4] considering only the transversal segmentation, not longitudinal, due to the cylindrical symmetry of the coaxial detector.

\section{Pulse generation and analysis}

The collection of the produced charge carriers has been calculated using the equation

$$
\frac{d q_{i}}{d t}=\frac{q_{i 0} \mu_{i}\left|\vec{E}\left(\vec{r}_{i}\right)\right|^{2}}{V_{0}\left(1+\left(\frac{E}{E_{\text {sat }}}\right)^{\beta}\right)^{1 / \beta}}
$$

The initial charge produced by the energy deposit $\left(\mathrm{q}_{\mathrm{i} 0}\right)$ moves along the detector following the electric field lines $(\overline{\mathrm{E}})$ producing a variation of the collected charge over the time $\left(\mathrm{dq}_{\mathrm{i}} / \mathrm{dt}\right)$. This variation also depends on the mobility of the charge carriers $\left(\mu_{\mathrm{i}}\right)$, the electric potential applied to the detector $\left(\mathrm{V}_{0}\right)$ and other parameters like $\mathrm{E}_{\text {sat }}$ or $\beta[5]$. 
The generated pulse is the representation of $\mathrm{dq}_{\mathrm{i}} / \mathrm{dt}$ during one microsecond sampled every nanosecond (see Fig. 1). The generated pulses have two singular points per energy deposit corresponding to the holes and electrons arrival. Due to the electric field topography, holes movement and arrival induce a parabolic shape in the pulse while the electrons produce a steplike function. In addition, since the electric field inside the detector only has radial component, the corresponding time of the singular points is only dependant on the radial position. For this reason the developed PSA only has radial sensitivity.

Based on the fact that double beta events have mostly single energy deposit, while background events frequently have more than one, the analysis is based on the determination of the pulse singular points. If a pulse has two singular points, it is supposed to be produced by one energy deposit, but if three or more singular points are detected, the event has two or more energy deposits and is rejected. The localization of the pulse derivative maxima corresponding to the singular points, is the procedure to determine the number of singular points (see Fig. 1).

An important issue for this pulse analysis is the time resolution. Two singular points could be close enough to entangle one each other if the experiment components or acquisition system have no enough time resolution, underestimating the number of singular points.

\section{Results}

Pulse analysis of events that leave all the energy in a single segment has been carried out for each of the background sources considered in section 2.1 taking into account different time resolution values between 5 and 100 nanoseconds for 2-kg and 4-kg germanium detectors considering initial background levels described in [1]. For all the configurations, ${ }^{60} \mathrm{Co}$ contamination in copper components is predominant having more than $90 \%$ contribution to the total background when a copper cryostat around $3 \mathrm{~kg}$ weight and $1 \mathrm{mBq} / \mathrm{kg}{ }^{60} \mathrm{Co}$ activity is considered; both mass and activity conditions could be improved. Figure 2 shows how the total background level increases for higher values of time resolution, a background rate of $10^{-3}$ $\mathrm{c} / \mathrm{keV} / \mathrm{kg} / \mathrm{y}$ seems to be unreachable even with good time resolution values. If the contamination coming from copper cryostat is not considered, the total background levels decrease reaching, for good time resolution values, the required background level. It is also important to note that final background level using $2-\mathrm{kg}$ or $4-\mathrm{kg}$ detectors is almost the same.
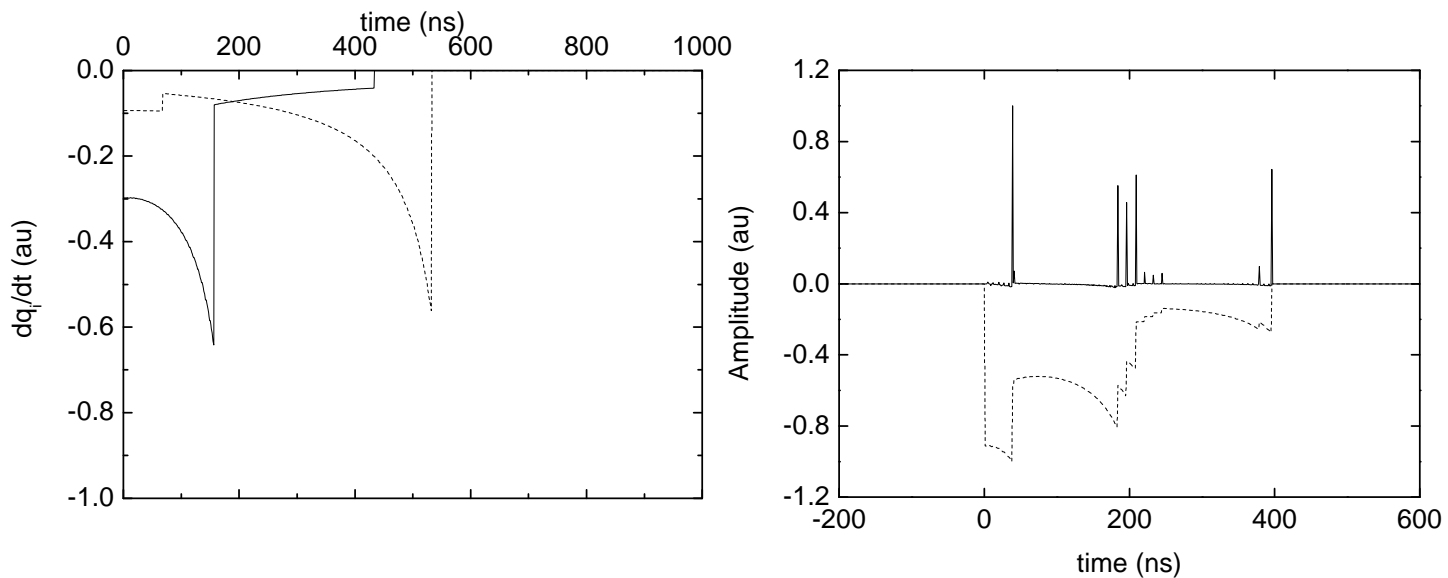

Figure 1: Left: Representation of two pulses originated by two single site events of the same energy at $18 \mathrm{~mm}$ (solid line) and $48 \mathrm{~mm}$ (dashed line) from the detector axis. Right: Representation of a pulse generated by an event with 5 energy deposits (dashed line) and its corresponding derivative (solid line). 


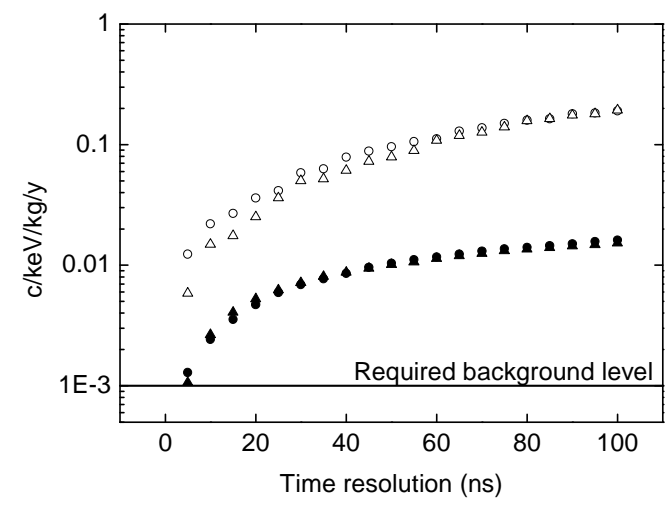

Figure 2: Representation of the background level in $\mathrm{c} / \mathrm{keV} / \mathrm{kg} / \mathrm{y}$ depending on the time resolution for a 2$\mathrm{kg}$ detector considering and not considering the contamination coming from the copper components (white and black triangles) and for a 4-kg detectors on the same conditions (white and black circles) The required background level considered is $10^{-3} \mathrm{c} / \mathrm{keV} / \mathrm{kg} / \mathrm{y}$.

\section{Conclusions}

PSA using segmented germanium detectors seems to be the most reliable tool to reach background and efficiency levels necessary to explore the neutrino effective mass region around $40 \mathrm{meV}$ investigating ${ }^{76} \mathrm{Ge}$ neutrinoless double beta decay. A full setup of calculations, including background events generation, electric field calculation inside segmented germanium detectors and routines to generate and analyze electric pulses generated from the background events, has been carried out trying to determine numerically the background level that could be reachable using this technique.

Taking into account some factors like time resolution or the initial background levels, a $10^{-3} \mathrm{c} / \mathrm{keV} / \mathrm{kg} / \mathrm{y}$ background level of seems to be unreachable using this technique unless contribution from copper components is kept under control. The development of a tridimensional PSA, considering the induced pulses in the adjacent segments to the one where the interaction occurs [6], could help to reach the required background level and is currently ongoing.

\section{Acknowledgments}

This work has been funded by Spanish MCyT/MEC contract FPA2004-0974 and the ILIAS integrating activity (Contract number: EU-RII3-CT2003-506222).

\section{References}

[1] H.Gómez et al, Astropart. Phys. 28 (2007) 435-447.

[2] S. Agostinelli et al, Nucl. Instr. and Meth. A 506 (2003) 250.

[3] S. Cebrián et al, J. of Phys.: Conf. Ser. 39 (2006) 344. Institute of Physics.

[4] http://WwW. comsol.com.

[5] G. Ottaviani et al, IEEE Trans. Nucl. Sci. NS-22 (1975) 192.

[6] Th. Kröll, D. Bazzacco, Nucl. Instr. and Meth. A 565 (2006) 691. 